\title{
Pag-uugat sa kultura ng pagkakasakit ng mga Taclobanon ng Hilagang Leyte
}

\author{
Amat, Aldwin B. $\triangle$
}

Leyte Normal University, Philippines (aldwinamat777@gmail.com)

Received: 25 May 2020

Available Online: 10 July 2020

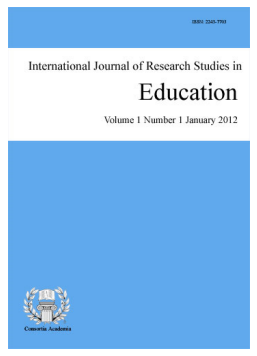

ISSN: $2243-7703$ Online ISSN: 2243-7711

OPEN ACCESS

\section{Abstract}

This study aimed to gather and analyze the beliefs related to the sickness of the Taclobanon in the Northern part of Leyte. This research also analyzed the words and cultural values found in the beliefs. The researcher used the ethnographic research design in gathering the beliefs related to the sickness of the Taclobanon. For the research locale, the mapping was conducted based on the different factors influencing the development of Filipino literature such as (a) remote areas (b) coastal areas (c) upland areas and (d) town centers in the province of Leyte. The researcher used purposive sampling in determining the informants of the study according to the following, (a) age: 55 years old and above, (b) was born and a resident of the place not less than fifteen years, and (c) a Taclobanon who is willing to share his/her knowledge about the beliefs related to the sickness of the Taclobanon. A semi-structured survey questionnaire was also used wherein the interview was open to all the responses of the informants. The results of the study showed that the common sickness of the Taclobanon was man-made by nature such as barang and hilo (poison). However, they believe that others were caused by supernatural beings known to them as kahuynon or engkanto (an enchanted being) such as ginbugkot (wherein human spirit has been carried away by an evil spirit), nahiungaran hin kataw (played by a mermaid), ginbuyong (lost by an evil spirit), and ginkakalagkalag (a belief in the presence of a dead person). In the cultural values particular in the physical dimension, it can be seen how people believe in nature's ability to treat illnesses such as the haro (known as a herbal plant) which is used in treating a particular illness, and beliefs in enchanted beings. In the spiritual dimension, the belief of herb doctors (known as albularyo) in God to heal people with illness, and the beliefs in other creatures such as supernatural beings was also shown in the results of the study.

Keywords: beliefs; values; barang; engkanteo; albularyo 


\section{Pag-uugat sa kultura ng pagkakasakit ng mga Taclobanon ng Hilagang Leyte}

\section{Introduksyon}

Ang Pilipinas ay nababalot ng maraming pamahiin at tradisyon ng matatanda tungkol sa mga bagay na walang relasyon sa ating nakikita o ginagawa. Halos bawat okasyon sa buhay ng tao ay may kaakibat na pamahiin na dapat sundin upang swertihin at malayo sa kapahamakan. Ito ay mga paniniwala na walang basehan kung ito ay may katotohanan o pawang nagkataon lamang (Soriano, 2015). Malaki ang naging impluwensiya ng mga pamahiin sa buhay ng mga Pilipino lalo na sa mga usapin sa buhay tulad ng kultura, emosyon, tagumpay at kabiguan. Ito ay isang kaugaliang namana sa ating mga ninuno na patuloy pa rin isinasagawa ng karamihan. Ayon sa nakakatanda, ang hindi pagsunod sa mga pamahiin ay nagdudulot ng kamalasan o ng kasawian.

Kaugnay sa pagkakasakit, ayon kay Alicio (2009) tungkol sa konsepto ng kulam sa sikolohiyang panlipunan sa Kabite, nabatid na ang kadalasang sanhi ng pagkakulam ay inggit o galit mula sa isang taong maalapit o kilala rin mismo ng biktima na binibigyan ng lunas sa pamamagitan ng mga dasal, ritwal o gamot na gawa ng isang albularyo o eksperto sa kulam. Karamihan ay naniniwala sa tradisyonal na panggagamot tulad na lang sa paggagamot sa lason at kulam. Kaugalian pa rin nila ang pagtayhup (pagbuga) at pagluon (pagluob) para sa mga karaniwang sakit. Binaggit sa pag-aaral ni Zafra (2010) sa tradisyonal na panggagamot sa Pagbilao, Quezon na ang paggagamot sa tuklaw ng ahas, pagbubuga ng balis, pangagamot ng lasom/ ugam, hilot at pagsuob ng taong nagalaw ay buhay na buhay pa rin sa nasabing bayan dahil na rin sa matinding paniniwala, usaping pinansiyal at komportableng pakikitungo ng mga albularyo ng Pagbilao.

May malalim din na pagtanaw ang mga taganayon ng Tacloban sa mga tambalan pinaniniwalaan nila na kapag hindi karaniwan ang sakit ng tao ay kagagawan ito ng masasamang elemento lalo na kung ito`y dinala sa ospital na hindi gumaling. Kaya karamihan ng mga taganayon na nakaranas dito ay naniniwala sa albularyo. Kaugnay nito, ang pagdarasal ng mga albularyo ng Pagbilao ay pamamaraan din na isinasagawa ng tambalan ng Leyte at Albularyo ng Batanggas. Sa katunayan, ayon kay Dr. Galvez Tan (1987), bukod sa pagiging mahusay na community health workers ng mga ito, ang tambalan ay hindi maikakailang sila ay may malalim na debosyon sa Panginoon.

Lumabas sa pag-aaral ni Timbreza (2008) na ang pakikisama ay karaniwang dahilan ng pagkakasakit ng tao ayon sa kanya na hindi lamang sa kapwa tao bagkus sa ibang nilalang na pinaniniwalaang nakatira sa ating kapaligiran. Sa isang pagtingin, maaaring ang suob bilang isang metodo ng panggagamot ay maiiugnay sa pakikisama at pagbibigay-galang sa mga ibang nilalang na nakatira sa paligid. Sa pagsusuri, maaaring ang mga ito ay pamamaraan din upang turuan ang tao na magbigay-halaga sa iba pang naninirahan sa kalikasan (Timbreza 2008). Maipapaliwanag din ito ng "mystical theory of illness" kung saan ang pagkakasakit ay produkto ng maling gawa ng tao. "Where health and illness are natural consequences of "good" and "evil" behavior, rather than as rewards or punishments from supernatural entity (Tan, 81). Samakatuwid, "manunumbalik" o tatalsik sa atin ang magiging bunga ng ating gawain at pag-uugali” (Timbreza, 2008).

Samantala, pinapahalagahan ang pagtatawas sa ilang lugar sa Pilipinas. Ayon kay Apostol (2010) ang tawas ay isang uri ng diagnosis o pagbasa sa karamdamang pisikal, astral, emosyonal, o mental ng isang tao. Ang tawas ay pamamaraan ng pagtuklas kung ang sanhi ng karamdaman ay masamang kulam. Natukoy sa pag-aaral ni Zafra (2010) na ang proseso ng gamutan sa Pagbilao Quezon ay naipakita ang pagpapahalagang espirituwal sa pamamagitan ng orasyon o bulong batay sa mga albularyo at mga kalahok ay malaki ang naitutulong nito sa kagalingan ng pasyente gayundin sa kanilang kapakanan bilang manggagamot.

Kaugnay nito, minabuti ng mananaliksik na pag-aralan ang mayamang kultura pagkakasakit ng mga Taclobanon kung paano ito pinahahalagahan tulad ng ilang katutubong Pilipino, ang pagkakaugnay-ugnay at pagkakaiba-iba nito sa ibang rehiyon. Mahalagang maidokumento ito sapagkat ito ang yaman na maaaring ipasa

30 Consortia Academia Publishing (A partner of Network of Professional Researchers and Educators) 
sa susunod na salinlahi, ang mga tradisyon at pamahiin na isa mga karunungang bayan na ipinakilala sa atin ng ating kanununan. Hindi rin maipagkakaila na ang mga pamahiin at tradisyon ay bahagi ng kulturang Pilipino.

\subsection{Balangkas teoritikal}

Sa teoryang ito ay nakasalig ang konsepto sa pagsusuri ng mga pamahiin at ang kaugnayan nito sa kulturang nakapaloob sa isang pamayanan. Pinatunayan ito sa pag-aaral ni Masendo (2015) hinggil sa mga kaugalian ng tribong Manobo ng Sitio Kapda, Brgy. Sab-ud, Loreto, Agusan del Sur, lumabas sa pag-aaral na ang mga ritwal, paniniwala sa espirito, pag-aasawa, pambubulong, pag-ayos ng alitan, paglilibing at pagtatalaga ng mga pinuo ay karaniwang kaugalian ng mga tribo. Naniniwala rin sila na ang mga pasalitang ritwal ay kinakailangan maisalimbibig upang ito`y hindi mamatay. Maliwanag na ang pamahiin at paniniwala ay bahagi ng identidad ng isang indibiduwal at mahalagang mailipat ang kaalamang ito sa paghubog sa katauhan ng isang katutubong mag-aaral.

Kaugnay nito ay sanligan din ang teoryang sosyolohikal na nagbibigay-diin sa interaksyon ng mga tao sa kapwa tao sa lipunan, ang sanhi ng pagkakaroon ng mga ganoong pangyayari, ang kanilang pakikibaka sa buhay, maging ang kanilang kabiguan o tagumpay. Ayon kina Villafuerte at Bernales (2009) sa isang pagsusuring sosyolohikal, tinitingnan ang kalagayan at ugnayan ng mga panlipunang institusyon tulad ng pamahalaan, simbahan, pamilya, paaralan at iba pa sa pagtatakda ng sitwasyon at oportunidad para sa mga mamamayan nito. Sa sosyolohikal na pananaw, mas malawak ang perspektib na pagsusuri ng isang akda. Hindi lamang ang kasiningan at naging katangian ng akda ang binubusisi, kundi pati na rin ang bahagi ng lipunan at kasaysayang pinagluwalan nito.

Ang bawat paglikha ng literatura, maituturing itong obra maestra ng may- akda. Masusi at maingat itong isinasalaysay at inilalarawan ang bawat damdamin, katulad ng matinding pag-ibig, pagkalungkot, pagkapoot, pagkatuwa, pagkasiya at iba pang maaaring maramdaman ng isang tao sa kanyang lipunang ginagalawan. Salamin din ang panitikan sa kulturang gumagana sa isang komunidad. Sa Pilipinas, bawat rehiyon ay mayaman sa tradisyon at kultura. Sa pamamagitan ng panitikan, nakikilala ang imahe ng bawat rehiyon. Hitik ito sa kani-kanilang makasaysayang ugat na patuloy na dumaloy at nabuhay sa pamamagitan ng kanilang mga bunga. Sa damdamin, kaisipan at mayamang karanasan ng indibiduwal ay nakabubuo siya ng pilosopiya, mga butil ng kaisipan, palaisipan, pamahiin, kwentong bayan, karunungang bayan at ritwal Rubin (2001). Bagay na nakasalig ito sa teoryang cultural relativism (Banggit ni Orobio, 2013), isinaalang-alang ang bawat kultura na kakaiba at natatanging yunit walang nakahihigit o mababa. Sinusuri sa panukat ang ito ang mga kaugaliang kultural saka isinasalang ang mga kaso na dapat hatulan kung tama o mali.

Pinagtibay ang `di matatawarang ugnayan ng kultura at wika sa "Sapir-Whorf hypothesis"na kombinasyong konseptong linguistic determinism na nagsasaad na hinuhubog ng wika ang paraan ng ating pag -iisip. May malaking kauganayan ito sa sinabi ni Eros Atalia (2008) na wika ang isang dahilan ng pagkakabuo ng mga pamahiin. "Isang tungkulin ng wika ang maipaliwanag ang realidad," aniya. "Nakatali sa wika ang limitasyon ng ating realidad at ng katotohanan." Hinuhubog ng wika ang kamalayan at kaugnayan sa daigdig at nagkakaroon din ng kolektibong pagtawag at konsepto ang mga bagay na nakapaligid sa atin tulad na lamang ng mga pamahiin. Ayon naman kay Arrogante (2010) na ang panitikan ng isang bansa ay sumasalamin sa mga paniniwala, kultura at tradisyon nito. Sa panitikan ibinubuhos ng mga tao ang kanilang mga saloobin, nararamdaman at nakikita sa kanilang katayuan kaya repleksyon ang panitikan ng kung ano ang pamumuhay sa isang bansa. Ang panitikan ay nagsasalaysay ng buhay, pamumuhay, lipunan, pamahalaan, pananampalataya at mga karanasang kaugnay ng iba't ibang uri ng damdaming tulad ng pag-ibig, kaligayahan, kalungkutan, pag-asa, pagkapoot, paghihiganti, pagkasuklam, sindak at pangamba. 


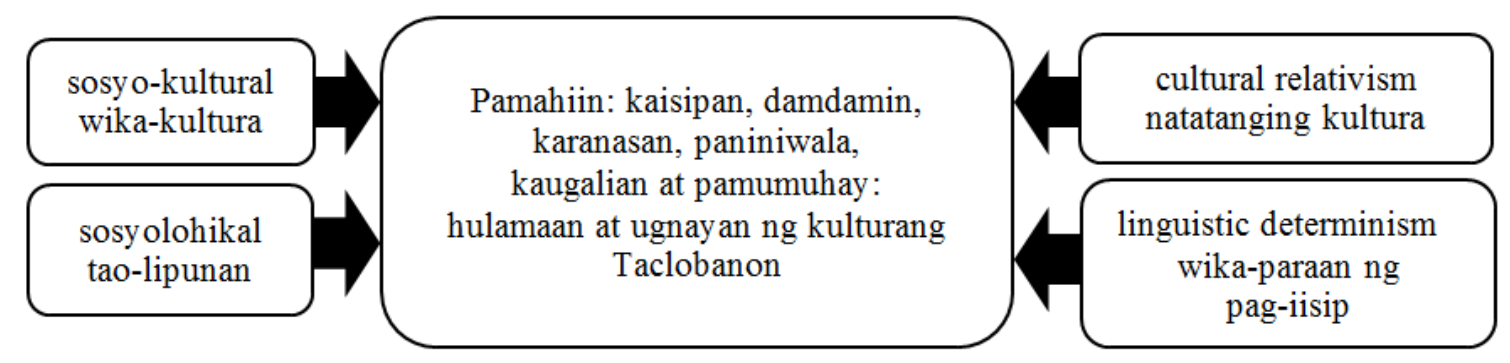

Figyur 1. Balangkas teoritikal ng pag-aaral

Nakasalig ang pamahiin sa kamatayan ng mga Taclobanon sa teoryang sosyo-kultural, sosyolohikal, cultural relativism at linguistic determinism. Ang mga kaisipan, damdamin, karanasan, paniniwala, kaugalian at pamumuhay ay masasalamin sa mga pamahiing sinusunod ng mga Taclobanon kaugnay sa kamatayan ng tao.

\subsection{Layunin ng pag-aaral}

Layunin ng pag-aaral na ito na makapangalap at masuri ang kultura pagkakasakit ilang piling barangay ng Lungsod Tacloban ng Hilagang Leyte. Upang maisakatuparan ang pag-aaral na ito, sisikaping sagutin ang mga sumusunod na mga katanungan:

$>\quad$ Ano-ano ang karaniwang pamahiin sa pagkakasakit ng mga Taclobanon ng Hilagang Leyte?

> Ano-ano ang pagpapahalagang kultural na nakapaloob sa pamahiin sa pagkakasakit ng mga Taclobanon ng Hilagang Leyte?

\section{Metodolohiya}

Ginamit ang desinyong etnograpiyang pananaliksik sa pangangalap ng mga pamahiin sa pagkakasakit ng mga Taclobanon. Saklaw sa gawaing ito ang panayam, pakikipagkwentuhan, obserbasyon, pagrirekord sa mga salaysay ng mga impormante na may malawak na kaalaman sa mga pamahiin sa kamatayan. Nasa purposive sampling ang paraan sa pagpili ng dalawang barangay na kumakatawan sa Lungsod Tacloban, ibinatay ito sa mga salik na nakaiimpluwensiya sa paglinang ng panitikang Pilipino gaya ng: (a.) liblib na lugar (b.) baybaying-dagat (coastal) (c.) kabundukan (upland) at (d) sentro ng bayan ng lalawigan ng Leyte.

Nasa paraang purposive sampling din sa pangangalap ng impormante na kung saan bukas ang mananaliksik sa pagkuha ng impormante depende sa kawilihan at sa panahong handang ilaan sa oras ng panayam. Pinagbatayan din sa pagkuha ng impormante ang sumusunod: (a.) edad 55- pataas (b.) ipinanganak at naninirahan sa komunidad na hindi bababa sa 15 taon (c.) inirekomenda ng residente at opisyal ng barangay na maalam sa mga pamahiin (d.) Taclobanon na handang magbahagi ng salimbibig na pamahiin na may kinalaman sa pagkakasakit. Semi-structured na talatanungan ang instrumentong ginamit sa pag-aaral na kung saan bukod sa mga tanong na makikita sa talatanungan ay bukas din ang mananaliksik at mga impormante sa mga karagdagang tanong at sagot sa mga pamahiing nabanggit sa oras ng panayam. Ibinatay ang sukatan sa pagpapahalagang kultural ni Ponsalan (1997) ng Values Education Program Framework ng DepEd na nahati sa pitong dimensyon: pisikal, espirituwal, sosyal, moral politikal, ekonomik at intelektuwal.

\section{Mga resulta at diskusyon ng pag-aaral}

Sa natural na pagkakasakit ng mga taganayon at pampang, lagnat ang isa sa mga karaniwang sakit ng kabataan at may ibinubulong ang matatanda sa karamdamang ito, tulad na lang sa pag-inom ng Royal (softdrinks). Bukod sa lagnat, karaniwan na sa matatanda ang sakit sa kasukasuan, mababatid sa pamahiing ito 
na ang pangunahing lunas nila ay pagluob ng butó ng kalabaw sa paniniwalang ito ang sanhi ng binat at ang tanging lunas lamang nito ay ang pagpapausok mula sa sinunog na butó ng kalabaw. Sa mga tagapampang, ang alternatibong gamot sa nanakit na kasukasuan ng matatanda ay ang paglubog ng paa sa buhangin habang naliligo sa dagat. Karaniwan na rin sa kabataan ang sakit sa balat na sapíl (pamamantal sa nguso) nakukuha ito sa mahilig na pag-amoy ng bata sa pagkain kaya sila nagkakasapíl karaniwan na ang pagtayhup (pagbuga sa ulo na may kasamang orasyon ng albularyo) ang bulong nito. Hindi rin maiiwasan ng mga taganayon at pampang ang makagat ng mga alupihan at isdang pagi dahil na rin sa kawalan ng proteksyon na isinusuot para sa kanilang katawan pinaniniwalaan ng matatanda na nakagagamot daw ang laway ng ari ng babae.

\section{Talahanayan 1}

Pamahiin sa pagkakasakit ng mga Taclobanon

\section{MGA PANADLIHAN HA PAGKAKASAKIT (MGA PAMAHIIN SA PAGKAKASAKIT)}

\section{A. Natural nga Sakit (Karaniwang Sakit)}

\section{1. hiranat (lagnat)}

Kun hitaas an imo hiranat, inom hin Royal kay napahabubo hin hiranat.

Kapag mataas ang lagnat, kailangan uminom ng Royal (softdrink gaya ng Coke) upang bumába ang lagnat.

\section{Lará Dara han Tinukban han Mananap (Kamandag Dala sa Kagat ng Insekto.}

Iginpapahid an laway han kanan babaye kasar-angan para panhimulong ha lará han tinukban han ulahipan ngan pagi. Ipinapahid ang laway na lumalabas mula sa ari ng babae upang gamutin ang kamandag na kagat ng alupihan at isdang pagi.

\section{Magpaol nga Kaluluthan (Nananakit na Kasukasuan)}

a. Dire puydi kumaon hin karne nga karabaw kun may balatian kay manmamaul iton imo kalawasan. Pananglit nahibughat, tungod han imo nakaon nga karne, ginpapaasuhan la an maul-ol nga dapit han lawas, gamit han binaga nga tul-an han karabaw.

Bawal kumain ng karneng kalabaw kapag nanánakit ang iyong katawan. Sakaling nagkabinat, kailangan mong pausukan ng sinunog na butó ng kalabaw ang tiyak na bahagi ng katawang nananakit upang gumaling ka.

b. Kun mag-ul-ol iton imo kaluluthan, iginlulubong la iton imo tiil ha baras han dagat kay nakakauli ini.

Kapag masakit ang iyong kasukasuan, kailangan mong ilibing ang iyong mga paa sa buhangin ng dagat, dahil nakapagpapagaling ito.

\section{4. sapíl (pamamantal)}

Napasapíl an mahilig mamaho hin pagkaon, mauupay la ini kun tatayhupan hiya hin tambalan o sarapilan.

Nagkakasapíl (pangangati at nanunubig na nguso at iba pang bahagi ng mukha) ang taong mahilig umamoy ng pagkain, gagalíng lang siya kapag tatayhupan (bubugahan sa ulo na may kasamang orasyon) ng albularyo.

5. Iba pa nga panadlihan ha pagkakasakit han tawo (iba pang panadlihan sa pagkakasakit ng tao)

Madali magkasakit an tawo nga iripahan hin sakit, mawawara la kun may-ada niya sul-ot nga relikyas o saót.

Madaling dapuan ng sakit ang taong lapitin ng sakit, mawawala lamang ito kapag may nakasabit sa kanya na relikyas.

\section{B. Mga Daot han Tawo (Kulam ng Tao)}

\section{1. barang (kulam)}

a. Kun may bakús (hinigot nga saót para diri ka dultan o tablan hin pandaot o barang) o kuwentas nga basiyo ha lawas dire ka tatablan hin daot o barang.

Kapag may may bakús (tali-taling panlaban sa kulam) o kuwentas na bala na nakatali sa iyong katawan ay hindi ka matatamaan ng daot o barang. 
Amat, A. B.

b. An tinikangan han sakit han tawo, kaurog natikang ha darahog han tawo ngan darahog han kahuynon.

Ang sakit ng tao, karaniwang nagmumula sa darahog (kulam) ng tao at darahog ng kahuyonon (kulam ng engkanto).

\section{2. hiló (lason)}

a. Mahisasabtan nga nahiluan ka kun haros waray kusog iton imo kaluluthan. Mahiuulian ka la kun papainumon ka hin tambalagisa ngan maisusuka mo kun ano an pagkaon nga nakahilo ha imo. Kaurog dire mo mahisasabtan kun hin-o an naghilo ha imo kay nahihinabo ini ha adlaw han panagkutan han mga patay, nahipapasa an hiló pinaagi hin pagtapik ha bayhon ngan paghuyop ha pagkaon

Malalaman na ika`y nalason kung walang lakas ang iyong kasukasuan. Magagamot lang ito kung papainumin mo siya ng tambalagisa (mapait at maliliit na botong gamot sa lason) saka maisusuka mo ang pagkaing nakalason sa`yo. Madalas ay hindi nalalaman kung sino ang nakalason dahil nagaganap ito sa araw ng paninidihan sa patay kung kailan matao ang iyong napuntahan, naipapasa ang lason sa pamamagitan ng pagtapik sa balikat at pag-ihip sa pagkain.

b. Kun may pangontra ka ha hiló, mahisasabutan kun an igindurut ha imo nga pagkaon o irimnun in mayda hiló, kay masukob o di ngani mabuto an lana nga imo iginbakús.

Kapag may panlaban ka sa lason, malalaman mo na ang iniabot sa`yo na pagkain o inumin ay may lason sapagkat kukulo o puputok ang langis na nakatali sa`yong baywang.

c. Kun may panguntra ka ha hiló, nalitík an baso nga imo ginkaptan kun ini in mayda hilo.

Kapag may panlaban ka sa lason, nabibitak ang basong iniaabot sa`yo.

\section{Mga Daot han Kahuynon (Kulam ng Masasamang Elemento)}

\section{Darahog han Kahuynon (naengkanto)}

a. Kun an usa nga tawo dire nahihiulian han hiranat ngan pirme la mag-ul-ol an iya kaluluthan, may posibilidad nga gindarahugan

hiya. Mabubulong la ini hiya kun maghalad hin irimnon ngan pagkaon kun diin hiya nga dapit nadarahugan, upód an paghudim-hudim hiton tambalan

Kapag hindi gumagaling ang taong may lagnat at sakit sa mga kasukasuan ay may posibilad na naengkanto siya. Magagamot lamang ito kapag mag-alay siya ng pagkain at inumin sa lugar na nadarahugan (napaglaruan ng masamang elemento) siya, kasabay sa pagsasagawa ng orasyon ng albularyo.

b. Kun an tawo gindarahugan, dire hiya mauupay bisan ano nga tambal an iya inumon. Makadto hiya ha tambalan basi hiya tayhupan o orasyunan

Kapag kinulam ang tao ay siguradong hindi siya gagaling anumang gamot ang kanyang inumin. Dinadala lamang siya sa albularyo upang magpatayhop (hipan sa ulo na sinasamahan ng dasal) at magpaorasyon upang gumaling siya.

c. Kun mag-inaringasa ka ha usa nga puy-aw nga lugar sugad ha umá o ha sapá, bangin hibuyagan ka hin engkanto

Kapag maingay ka isang lugar na tahimik gaya sa palayan, bukid o sa sapa, `di malayong maengkanto ka.

d. Dire puydi magpaaga hin sinampay ha gawas kay bangin pag-among-amongan ka hiton mga kahuynon, nga amo an magin hinungdan han imo kasakit. 
Bawal paabutan nang umaga ang iyong mga isinampay, baka ito`y paglaruan ng masasamang elemento, na siyang maging dahilan ng iyong pagkakasakit.

e. Kun mag-uma ka nga waray panabì ha mga kahuynon, dadarahugan ka.

Kung magsaka ka na walang pahintulot sa mga engkanto, sasaktan ka.

f. Kun nawarayan pamurubuot an usa nga tawo, hiya in gindarahugan, gin-among-amungan hiton mga kahuynon.

Kapag nawalan ng katinuan ang isang tao, naengkanto siya, kinukulam ng masasamang elemento.

\section{2. ginbugkot (dinala ang kaluluwa ng masamang espiritu sa kabilang mundo)}

An tawo nga ginbugkot o waray na pakagmatá in ginsaniban an lawas hin maraot nga espiritu, gindara iton iya espiritu han panulay nga amo an hinungdan han iya madagmit nga kamatayon.

Kapag binugkot (dinala ng masamang espiritu sa ibang mundo) o hindi na nagising ang tao, may sumanib sa kanyang katawan na masamang espiritu, dinala ang kanyang espiritu ng masamang elemento na siyang naging dahilan ng kanyang maagang kamatayan.

\section{3. ginbuyong (niligaw ng masamang espiritu)}

a. Kun ginbubuyong ka, kinahanglan baliktaron nimo an imo bado, basi hitultol ka han imo kakadtuan.

Kapag naliligaw ka, kailangan mong baliktarin ang iyong damit upang matunton mo ang iyong pupuntahan.

b. Kun maghuni an tigkaró dida ha iyo bungsaran, nambabaló (nanhahadlok) o nanhahadlok ini hiya.

Kapag humuni ang tigkaró (Isang ibon na may malaking mata na kulay kayumanggi, humuhuni lamang kapag hapon) sa inyong bakuran, tinatakot ka nito

\section{4. ginkakalág-kalág (pagpaparamdam ng patay)}

a. Kun mag-inop ka hin mga patay nga imo nakaistorya dida ha iyo panimalay, kinahanglan ka magpapangadi kay pagkakalag-kalagon ngan magkakasakit ka.

Kapag managinip ka ng mga taong namayapa na at nagkausap-usap kayo sa bahay mo, kailangan mong mag-alay ng dasal, dahil mumultuhin ka na siyang ikasasakit mo.

b. Kun makabaho ka hin kandila, gin-uungaraan ka hiton mga kalag nga amo an hinungdan nga naiirapa an tawo, kinahanglan hiya magpapangadi ngan maghalad hin pagkaon hadton mga nawara, basi hiya dire pagkalagon.

Kapag makaamoy ka ng usok-kandila, may hinihiling sa`yo ang kaluluwa ng patay na siyang naging dahilan ng iyong pagkakasakit. Kailangan mong magpadasal at mag-alay ng pagkain sa mga namayapa na upang hindi ka pagkalagon (paramdamin ng taong patay na).

c. Kun namatayan ka, kinahanglan ka maghalad (pagkaon, pamisa ug pag-ampo) basi dire ka pagmultuhon.

Kapag namatayan ka, kailangan mong mag-alay (pagkain, magpamisa, o panalangin) sa patay para hindi ka multuhin.

\section{Nahiungaraan han Katáw (Nagustuhan ng Sirena)}

Kun hibuyagan ka hin katáw in magkakairapa ka, kinahanglan nga maghalad ka hin pagkaon ha dagat basi ka hiulian.

Kapag nagustuhan ka ng sirena ay magkakasakit ka, kailangan mong mag-alay ng pagkain sa dagat para gumaling ka.

\section{Santilmo (nagpapakitang apoy na bigla na lamang nawawala)}

Kun makakaita ka hin santilmo ha dagat kinahanglan mo labayon ini hin tal-is nga dinagkutan para mahisumpa ini.

Kapag makakita ka ng santilmo sa dagat kinakailangan mong magtapon ng palitong nag-aapoy upang mawala ang sumpa. 
Sa kulam na gawa ng tao, karaniwan nang karamdaman ang lason at kulam ng mga taganayon at pampang. Kadalasan ang matatanda ay nagsusuot ng anting-anting bilang panlaban sa kulam at lason para hindi sila mapahamak tuwing dadalo sa mga handaan. Samantala, marami naman ang naitalang pamahiin na mula sa kulam ng masasamang elemento. Nauuri ang katawagang ito ayon sa kontekstong lokal, para sa mga taganayon ang nangkukulam na masasamang elemento ay kahuynon o engkanto samantala sa pampang ay katáw o sirena. Kadalasan, nakukuha ang kulam ng masasamang elemento sa hindi pagbibigay-galang ng mga taganayon at pampang tulad na lamang sa pag-iingay sa liblib na lugar, pagpuputol ng punongkahoy na walang paalam at pagtatrabaho sa bukid tuwing tanghali, upang hindi sila maengkanto ay humihingi sila ng pahintulot bago putulin ang puno sapamamagitan ng pag-alay nito ng pagkain. Iniiwasan din na mag-ingay sa mga liblib na lugar sa paniniwalang may nakatira na mga elementong 'di nakikita na may kakayahang makapanakit sa tao. Samantala, sa mga tagapampang naman naniniwala sila na ang `di karaniwang karamdaman ng tao ay kagagawan ng sirena kaya inaalayan nila ito ng pagkain upang gumaling ang may karamdaman.

Makikita rin dito ang karaniwang palatandaan ng mga taganayon at pampang kapag ang tao ay nakulam o sinaktan ng masasamang elemento, tanging albularyo lang ang makapagpapagaling nito. Kadalasan ang hindi paggaling ng lagnat, sakit sa kasukasuan at pagkabaliw ay isang anyo ng darahug o kulam ng engkanto. Maliban sa naengkanto, may iba-iba ring paraan kung paano manakít ang masasamang elemento, karaniwan na sa mga taganayon at pampang ang bugkót (dinala ang kaluluwa ng masamang espirtu). Maliban sa bugkót, ang buyong ay isa ring paraan sa panghahamak ng masasamang elemento ayon sa mga impormante, nililigaw ka nito na hindi ka makarating sa iyong pupuntahan at masusumpa lamang ito kapag babaligtarin mo ang iyong kasuotan. Naniniwala rin ang mga mangingisda tungkol sa santilmo, pinaniniwalaan nila na ang santilmo raw ay ligaw na kaluluwang nagpapakita sa ibabaw ng tubig sa dagat.

Bukod sa masasamang elemento, nagpaparamdam din ang kaluluwa ng patay sa mga taong mahal niya sa buhay sakaling hindi ito naalayan ng dasal. Ito rin ang itunuturong dahilan sa pabalik-balik na pagkakasakit ng mga tao sa kanayunan o ginkalagkalag kadalasan inaalayan ng dasal, misa at pagkain ang taong namayapa na upang mapakali ang kanyang kaluluwa.

Malinaw na ang mga karaniwang karamdaman na nakukuha ng mga taganayon at pampang ng Tacloban ay lagnat, sakit sa kasukasuan, kagat ng alupihan, sapil at iba pa. Ang sakit na kagagawan naman ng tao ay barang o kulam at hiló o lason. Samantala, ang kulam naman na gawa ng masasamang elemento ay naengkanto, bugkot (dinala ang kaluluwa ng tao ng masamang espiritu), buyong (niligaw ng masamng espiritu), kalág-kalág (pagpaparamdam ng patay). Sa mga tagapampang ay pinaniniwalaan naman ang napaglaruan ng sirena. Nabatid din sa pag-aaral na ito na madalas puntahan ng mga taganayon at pampang ay tambalan o albularyo.

\section{Talahanayan 2}

Pagpapahalagang kultural sa pamahiin sa pagkakasakit

\begin{tabular}{|c|c|}
\hline Pamahiiin & Pagpapahalagang Kultural \\
\hline \multicolumn{2}{|c|}{ Dimensyong Politikal at Pisikal } \\
\hline Ipinapahid ang laway na lumalabas mula sa ari ng babae upang gamutin ang & Pananalig sa kakayahan ng kababaihan. \\
\hline kamandag na kagat ng alupihan at isdang pagi. & Paggalang sa kasarian. \\
\hline $\begin{array}{l}\text { Kapag mataas ang lagnat, kailangan uminom ng Royal (softdrink gaya ng Coke) } \\
\text { upang bumába ang lagnat. }\end{array}$ & Pakikiuso \\
\hline
\end{tabular}
upang bumába ang lagnat.

Pagsunod sa tradisyonal na pangagamot. Pananalig sa albularyo.

Pananalig sa bisa ng relikyas. Panaling panlaban sa kulam.

Pananalig sa bisa ng relikyas o panaling panlaban sa kulam. 
Talahanayan 2 ...pagtutuloy

\begin{tabular}{|c|c|}
\hline Pamahiiin & Pagpapahalagang Kultural \\
\hline $\begin{array}{l}\text { Ang sakit ng tao, karaniwang nagmumula sa darahog (kulam) ng tao at darahog } \\
\text { ng kahuyonon (kulam ng engkanto). }\end{array}$ & Paniniwala sa kulam ng tao at engkanto. \\
\hline Malalaman na ika`y nalason kung walang lakas ang iyong mga kasukasuan. & Paniniwala sa lason. \\
\hline Magagamot lang ito kung papainumin ng tambalagisa (mapait at maliliit na & Pananalig sa halamang gamot. \\
\hline $\begin{array}{l}\text { botong gamot sa lason) saka maisusuka ang pagkaing nakalason. Madalas ay } \\
\text { hindi malalaman kung sino ang nakalason dahil nagaganap ito sa araw ng } \\
\text { paninidihan sa patay kung kailan matao ang iyong napuntahan, naipapasa ang } \\
\text { lason sa pamamagitan ng pagtapik sa balikat, pag-ihip sa pagkain. }\end{array}$ & Pag-iingat sa pakikitungo sa mga taong `di kilala. \\
\hline Kapag may panlaban ka sa lason, malalaman mo na ang iniabot sa yo na pagkain & Paniniwala sa lason. \\
\hline o inumin ay may lason sapagkat kukulo o puputok ang langis na nakata sa yong & Pananalig sa bisa ng langis. \\
\hline baywang. & Pag-iingat sa pakikitungo sa mga taong `di kilala. \\
\hline Kapag may panlaban ka sa lason, nabibitak ang basong iniaabot sa`yo na may & Paniniwala sa lason. \\
\hline lason. & Pananalig sa panlaban sa lason. \\
\hline $\begin{array}{l}\text { Kapag hindi gumagaling ang taong may lagnat at sakit sa kasukasuan ay may } \\
\text { posibilad na naengkanto siya. Magagamot lamang ito kapag mag-alay siya ng }\end{array}$ & $\begin{array}{l}\text { Paniniwala sa darahug o kulam ng masasamang } \\
\text { elemento. }\end{array}$ \\
\hline $\begin{array}{l}\text { pagkain at inumin sa lugar na nadarahugan(napaglaruan ng masamang elemento) } \\
\text { siya, kasabay sa pagsasagawa ng orasyon ng albularyo }\end{array}$ & Pananalig sa orasyon. \\
\hline Kapag kinulam ang tao ay siguradong hindi siya gagaling anumang gamot ang & Pananalig sa albularyo. \\
\hline $\begin{array}{l}\text { kanyang inumin. Dinadala lamang siya sa albularyo upang magpatayhop (hipan } \\
\text { sa ulo na sinasamahan ng dasal) at magpaorasyon upang gumaling siya. }\end{array}$ & Paniniwala sa kulam. \\
\hline $\begin{array}{l}\text { Kapag maingay ka isang lugar na tahimik gaya sa palayan, bukid o sa sapa, ’di } \\
\text { malayong maengkanto ka. }\end{array}$ & Paniniwala sa engkanto. \\
\hline $\begin{array}{l}\text { Bawal abutan nang umaga ang mga isinampay, baka ito`y paglaruan ng } \\
\text { masasamang elemento, na siyang maging dahilan ng iyong pagkakasakit. }\end{array}$ & Paniniwala sa engkanto. \\
\hline Kung magsaka ka na walang pahintulot sa mga engkanto, sasaktan ka. & Paniniwala sa engkanto \\
\hline $\begin{array}{l}\text { Kapag nawalan ng katinuan ang isang tao, naengkanto siya, kinukulam ng } \\
\text { masasamang elemento. }\end{array}$ & Paniniwala sa kulam ng masasamang elemento. \\
\hline Kapag binugkót (dinala ng masamang espiritu sa ibang mundo) o hindi na & Paniniwala sa bugkót. \\
\hline $\begin{array}{l}\text { nagising ang tao, may sumanib sa kanyang katawan na masamang espiritu, dinala } \\
\text { ang kanyang espiritu ng masamang elemento na siyang naging dahilan sa } \\
\text { kanyang maagang kamatayan. }\end{array}$ & Paniniwala sa masasamang elemento. \\
\hline $\begin{array}{l}\text { Kapag naliligaw ka, kailangan mong baliktarin ang iyong damit upang matunton } \\
\text { mo ang iyong pupuntahan. }\end{array}$ & Paniniwala sa masasamang elemento. \\
\hline $\begin{array}{l}\text { Kapag humuni ang tigkaró (Isang ibon na may malaking mata na kulay } \\
\text { kayumanggi, humuhuni lamang kapag hapon) sa inyong bakuran, tinatakot kayo } \\
\text { nito. }\end{array}$ & $\begin{array}{l}\text { Paniniwala sa mga nilalang pinamamagitan ng } \\
\text { masasamang elemento. }\end{array}$ \\
\hline $\begin{array}{l}\text { Kapag managinip ka ng mga taong namayapa na at nagkausap-usap kayo sa } \\
\text { bahay mo, kailangan mong mag-alay ng dasal, dahil mumultuhin ka na siyang } \\
\text { ikasasakit mo. }\end{array}$ & $\begin{array}{l}\text { Paniniwala sa pagpaparamdam ng taong namayapa. } \\
\text { Pananalig sa dasal. }\end{array}$ \\
\hline $\begin{array}{l}\text { Kapag makaamoy ka ng usok-kandila, may hinihiling sa`yo ang kaluluwa ng } \\
\text { patay na siyang naging dahilan ng iyong pagkakasakit. Kailangan mong } \\
\text { magpadasal at mag-alay ng pagkain sa mga namayapa na upang hindi ka } \\
\text { pagkalagón (paramdamin ng taong patay na). }\end{array}$ & $\begin{array}{l}\text { Paniniwala sa pagpaparamdam ng taong namayapa. } \\
\text { Pananalig sa dasal }\end{array}$ \\
\hline $\begin{array}{l}\text { Kapag namatayan ka, kailangan mong mag-alay (pagkain, magpamisa, o } \\
\text { panalangin) sa patay para hindi ka multuhin. }\end{array}$ & $\begin{array}{l}\text { Paniniwala sa pagpaparamdam ng taong namayapa } \\
\text { at pananalig sa dasal }\end{array}$ \\
\hline $\begin{array}{l}\text { Kapag nagustuhan ka ng sirena ay magkakasakit ka, kailangan mong mag-alay ng } \\
\text { pagkain sa dagat para gumaling ka. }\end{array}$ & Paniniwala sa masasamang elemento. \\
\hline
\end{tabular}

Nabibilang sa dimensyong politikal at pisikal ang pagpapahalaga sa pananalig sa kakayahan ng kababaihan. Dimensyong politikal sapagkat makikita rito ang kaugnayan sa kakayahan ng babae sa makamandag na insekto, isda at iba pa na hindi makikita sa kakayahan ng lalaki, minsan iniuugnay ang pananaw na ito sa Inang Kalikasan o Mother Earth. Nabibilang din ito sa dimensyong pisikal sapagkat pinapahalagahan dito ang kalusugang pangkatawan ng mga taganayon at pampang sa pamamagitan ng pagbulong sa tuklaw ng alupihan at isdang pagi.

Mababatid naman dito ang pagpapahalaga sa pagtatangi sa uso ng mga tagapampang na makikita sa pamahiing ito na "Kapag mataas ang lagnat, kailangan uminom ng Royal (softdrink gaya ng Coke) upang bumába ang lagnat "ayon sa impormante kaya Royal ang ipinaiinom sa may lagnat dahil mataas ito sa bitamina $\mathrm{C}$ at ito rin ang ipinasasalubong nila sa ospital tuwing dadalaw sila sa kanilang kaanak na may sakit.

Nauuri naman sa dimensyong pisikal ang mga pagpapahalagang ito sa pangangalaga sa kalusugang pangkatawan at ang pananalig ng mga taganayon at pampang sa tradisyonal na paraan ng panggagamot. Nauugnay rin sa tradisyonal na panggagamot ang malalim na pananalig ng mga taganayon at pampang sa albularyo na nabibilang sa dimensyong espirituwal, ang mga orasyon at dasal na pinaniniwalaang nakagagamot sa karaniwang karamdaman ng tao, maging ang mga karamdaman na kagagawan ng mga nilalang na hindi 
Amat, A. B.

nakikita at tao katulad na lamang sa kulam at lason.

Nakaugat na sa kulturang Pilipino ang pananalig sa kakayahan ng mga albularyo, madalas orasyon at pagbugá ang pamamaraan ng kanilang panggagamot. Sa mga taganayon at pampang ng Tacloban ay malakas ang paniniwala nila rito kahit sa sentro ng lungsod. Ayon sa mga impormante, tinatangkilik nila ang tradisyonal na paraan ng panggagamot sapagkat mura ang gastos dito lalo na sa mga mahihirap na hindi kayang magpaospital. May ilan naman na sumasadya sa albularyo kahit may pera dahil malakas ang kanilang pananalig sa kulam. Sa pag-aral na ito, lumabas na tinatangkilik ng mga taganayon at pampang ng Tacloban ang pangagamot sa albularyo dahil sa aspetong pinansiyal at sa malalim pananalig sa kakayahan ng tambalan.

Bahagi rin ng pagpapahalagang kultural ng mga Pilipino ang paniniwala ng mga tao sa mga hindi nakikitang nilalang, na nabibilang sa dimensyong espirituwal na madalas idinadahilan ng mga taganayon at pampang na ang hindi paggaling ng sakit at ang pagkabaliw ng tao ay kagagawan ng mga engkanto at iba pang masasamang elemento. Nabanggit sa mga naunang pag-aaral na ang mga Pilipino ay may malalim na paniniwala sa mga espiritung hindi nakikita, may malaking kaugnayan ito sa mga taganayon at pampang ng Tacloban, mababatid na maraming tambalan sa kanayunan ang madalas nilalapitan ng mga tagabaryo tuwing may karamdaman ang kanilang kaanak, madalas kinulam o naengkanto ang mga ito. Mapapansin din sa kanilang usapan na kahit karaniwang karamdaman lamang ay palaging iniuugnay sa naengkanto o napaglaruan ng espiritu at kung ano pa.

Nauugnay rin sa dimensyong sosyal ang paniniwala ng mga Taclobanon sa hiló o lason, ayon sa mga impormante, madalas nalalason ang taong dayo o hindi kaya ang kaanak o kamag-anak na hindi maganda ang pakikitungo sa kanila. Ayon sa mga impormante, kadalasan nalalason ang tao sa mga tagayan o inuman, piyesta at panagkutan (Mga araw na sinusubuk ang bisa ng kapangyarihan ng tambalan). Nalalason ang tao dahil sa nakursunadahan ito o hindi kaya, inggit at malalim na galit ng mga taong malapít sa`yo. Sa mga tagabaryo, mapapansin na makikita pa ang ganitong paniniwala dahil sa mga paalala ng mga kaanak na huwag basta-basta bibili at iinom o kahit sa pakikipag-usap sa taong hindi kilalá. Mapapansin din sa mga tagabaryo na may isinasabit na basyo ng bala, relikyas, lana at iba pa na panlaban sa kulam ng tao. Sa pagsunod sa pamahiing ito ay makatutulong ito sa mga tagabaryo na mag-ingat sa kanilang mga sarili, huwag basta-basta magtitiwala sa iba at magbigay-galang sa mga kamag-anak at sa ibang tao. Nabibilang din sa dimensyong espirituwal ang pagpapagahalaga ng mga taganayon at pampang sa kaluluwa ng taong namayapa na, kaya inaalayan nila ito ng dasal at misa upang matahimik ang kaluluwa ng yumao.

\section{Konklusyon at rekomendasyon}

Napatunayan sa pag-aaral na ito na ang sumusunod na:

> Mataas ang paniniwala ng mga taganyon at pampang ng Tacloban na ang nakukuha ang sakit ng tao mula sa kulam na gawa ng tao tulad ng lason at barang. Nakukuha rin ito sa kulam ng masasamang elemento tulad na lamang sa naengkanto, nabugkot, ginkalagkalag, nabuyong. Samantala, ang sapil, lagnat at pananakit sa kasukasuan ang karaniwang sakit ng mga taganayon at pampang ng Tacloban.

$>\quad$ Mababatid ang mga pagpapahalagang kultural na hango sa pamahiin ng pagkakasakit tulad na lamang sa pananalig ng mga tagabaryo sa kakayahan ng albularyo. Mataas na paniniwala sa Diyos ng mga albularyo na nabibilang sa dimensyong espirituwal. Nabibilang naman sa dimensyong pisikal ang pananalig ng mga tagabaryo sa bulong ng mga halamang gamot, ang mataas na pagpapahalaga sa kalusugan. Nauugnay rin sa dimensyong ekonomiko ang pagpapagamot ng mga tagabaryo sa tambalan dahil sa kakapusan ng pera.

Inererekomenda sa pag-aaral na ito ang sumusunod na:

> Magsagawa pa ng mga pag-aaral tungkol sa paglilikom ng mga salaysayin para sa preserbasyon at pagpapayaman ng panitikang lokal.

38 Consortia Academia Publishing (A partner of Network of Professional Researchers and Educators) 
Pag-uugat sa kultura ng pagkakasakit ng mga Taclobanon ng Hilagang Leyte

$>\quad$ Isama ang nabuong pag-aaral sa pagtuturo ng panitikang rehiyonal bilang tugon ng Kagawaran ng Edukasyon sa lokalisasyon at integrasyon ng mga babasahing lokal at rehiyonal.

\section{Sanggunian}

Alicio, M. T., Donor, R. M., Jeresa G., A. M., \& Mijares, A. B. (2009). Ang konsepto ng kulam sa sikolohiyang panlipunan sa Kabite [Undergraduate thesis]. De La Salle University-Dasmarinas.

Apostol, V. M. (2010). Way of the ancient healer: Sacred teachings from the Philippine ancestral traditions. Berkeley, California.

Arrogante, J. A., Ayuyao, N. G., \& Lacanlale, V. M. (2010). Panitikang Filipino antolohiya binagong edisyon. Mandaluyong City: National Book Store.

Atalia, E. (2008). Orakulo ng kapalaran ni M. J. Enriquez. Retrieved from http://varsitarsitarian.net//filipino/20081117/mensahe_ng_pamahiin

Masendo, A. L. (2015). The Manobo tribe then and now: An ethnography. International Journal of Scientific and Engineering Research, 6(4). Retrieved from https://www.ijser.org/paper/The-Manobo-Tribe-Then-and-Now-An-Ethnography.html

Orobia-Asaria, J. A. C. (2013). Ang materyal at `di materyal na kulturang tribong Manobo sa Don Carlos, Bukidnon. Published Research. Central Mindanao University Musuan, Maramag.

Orobio (2013). Cultural relativism. Retrieved from http://www.chegg.com/homeworkhelp/definitions/cultural-relativism-49

Punsalan, T. (1997). Curriculum and instruction: Edukasyon sa pagpapahalaga at edukasyong pagpapakatao. Values Education Program (VEP) Framework. DepEd.

Rubin, L. T. (2001). Ang sining ng tula at maikling kwentong pambata. Manila: Rex Book Store.

Semorlan, T. P., Semorlan, A., Cañete-Mariño, F., Fernandez-Cabaron, E., \& Antonio, L. F. (2014). Ang panitikan at kulturang Pilipino. Quezon City: C\& E Publishing Company.

Soriano, R. (2015). Ang mga paniniwalang taglay ng mga Pilipino. Retrieved from https://uclaliwanagatdilim2015.wordpress.com/2015/03/06/1302/

Tan, M. (1987). Usug, kulam, pasma: Traditional concepts of health and illness in the Philippines. Alay Kapwa Kilusang Pangkalusugan.

Timbreza, F. (2008). Philosophy of life and values: Filipino philosophy today. Diliman, Quezon City: National Book Store.

Zafra, R. G. (2016). Suob, buga, sikolohiya: Isang pag-aaral sa kultura ng tradisyunal na panggagamot sa Pagbilao, Quezon. Manila: De La Salle University. 
Amat, A. B.

40 Consortia Academia Publishing (A partner of Network of Professional Researchers and Educators) 